\title{
Efeitos da independência do banco central e da taxa de rotatividade sobre a inflação brasileira
}

\author{
HELDER FERREIRA DE MENDONÇA*
}

The effects of central bank independence and the rate of turnover on the Brazilian inflation. Nowadays, the belief is strong in the Brazilian federal government that operational central bank independence is a basic condition to assure price stability. The literature concerning this subject highlights that a high degree of independence and a low turnover of central bank governor are associated with low inflation. This paper analyzes the relation between central bank independence and inflation in Brazil during 1980-2002. The findings denote that there is no effect on inflation due to an increase in degree of independence and a fall in turnover rate.

Key-words: central bank independence, inflation, turnover of central bank governors.

JEL classification: E58.

\section{INTRODUÇÃO}

O debate acadêmico iniciado no final dos anos 1970 intitulado "regras versus discrição" tem influenciado em grande medida a análise sobre a credibilidade da política econômica. Desde a segunda metade dos anos 1980 tem se tornado cada vez mais numeroso os defensores da idéia de que uma elevada credibilidade, proveniente da adoção de um banco central independente, é capaz de reduzir e manter a inflação estável tendo como contrapartida um baixo custo social. ${ }^{1}$

\footnotetext{
*Professor do Departamento de Economia da UFF e pesquisador do CNPq. E-mail: helderfm@hotmail.com. Agradeço aos profícuos comentários realizados por um parecerista anônimo desta Revista. Possíveis erros e omissões são de exclusiva responsabilidade do autor. Submetido: janeiro 2004; Aceito: maio 2005.

${ }^{1}$ Alesina e Summers (1993) representam um bom exemplo daqueles que acreditam que independência do banco central promove estabilidade de preços e não implica efeitos reais significativos sobre a economia. Entretanto, nem todos partilham dessa visão. Posen (1998) encontra evidências de que não há um custo de desinflação menor para países com bancos centrais independentes.
} 
Blinder (2000) mostrou, por meio de um questionário submetido a diversos representantes de banco centrais e economistas, que uma das condições mais importantes apontada por esses profissionais para estabelecer ou manter a credibilidade é a independência do banco central. ${ }^{2}$ Sob essa perspectiva, diversos artigos têm recomendado a adoção de um banco central independente com o objetivo de inibir dois problemas relacionados à condução da política monetária: (i) a presença do viés inflacionário em políticas discricionárias; e (ii) o uso político da política monetária. ${ }^{3}$

De acordo com a Constituição de 1988, no seu artigo 164, o Banco Central do Brasil é proibido de financiar, de forma direta ou indireta, o Tesouro Nacional e qualquer entidade que não seja financeira. Assim, dado que a maior parte dos economistas interpreta o fato de o banco central não monetizar déficits orçamentários como uma das mais importantes características de independência da autoridade monetária, observa-se que a lei imposta pela Constituição de 1988 representa um avanço para aumentar a independência do banco central. A partir da introdução do Plano Real as discussões políticas sobre a possibilidade da adoção de um banco central independente para a economia brasileira afloraram. No período recente $o$ governo Lula tem ressaltado que a independência operacional do banco central representa condição sine qua non para assegurar a estabilidade de preços.

O termo "independência operacional" é derivado da idéia do que Fischer (1995) batizou de independência de instrumento (instrument independence). De acordo com essa perspectiva, uma das condições para que o banco central seja dotado de independência é a necessidade de ter à sua disposição os instrumentos necessários para alcançar seus objetivos sem depender de nenhuma outra autoridade política. Em outras palavras, a independência operacional do banco central pressupõe a livre utilização de instrumentos de política econômica para a busca da estabilidade de preços. Conforme ressaltado por Goodhart (1994) a fundamentação teórica para a independência do banco central tem como base dois conceitos: (i) a ausência de trade-off entre a inflação e o desemprego no longo termo, isto é, a validade de uma curva de Phillips vertical; e (ii) o conceito do ciclo político de negócios. ${ }^{4}$

"A análise empírica sobre independência do banco central e inflação se notabilizou por revelar uma relação inversa entre essas variáveis. É reconhecido pela literatura que há grande dificuldade em quantificar de forma sistemática os fatores formais e informais responsáveis pela

\footnotetext{
${ }^{2}$ Apenas a história de honestidade da instituição recebeu maior importância do que a independência do banco central.

${ }^{3}$ Para uma análise pormenorizada desses dois pontos, ver Mendonça (2002).

${ }^{4}$ Uma boa resenha da literatura sobre independência do banco central pode ser encontrada em Berger, de Haan e Eijffinger (2001), e de Mendonça (2000).
} 
independência do banco central (Cukierman, 1996). Na grande maioria das vezes estes resultados foram obtidos a partir de indicadores baseados nas leis dos bancos centrais. ${ }^{5} \mathrm{O}$ principal problema com esse método é que, na maioria das vezes, ele não é capaz de capturar as transformações de variáveis econômicas ocorridas em um país. Além disso, há consenso de que indicadores legais são menos úteis para países em desenvolvimento" (de Haan e Kooi, 2000).

Em uma tentativa de atenuar o problema supracitado, Cukierman (1992) e Cukierman, Webb e Neyapti (1992) desenvolveram um índice que leva em consideração um questionário submetido para funcionários qualificados de diversos bancos centrais com o objetivo de avaliar a divergência entre a prática e o que é estabelecido em lei. Em outras palavras, esse índice tem como referência julgamentos sobre o comportamento da autoridade monetária.

Um ponto que merece destaque na literatura sobre a independência do banco central, no caso de países em desenvolvimento, refere-se ao número de vezes que o presidente do banco central é substituído durante o mandato do Presidente da República. De acordo com Cukierman, Webb e Neyapti (1992) a rotatividade dos presidentes do banco central serve como um indicador que é capaz de dar uma noção mais realista para a independência dos bancos centrais por permitir uma avaliação do comportamento de fato do banco central.

"The idea behind this measure is that, even if the central bank law is quite explicit, it may not be operational if a different tradition has precedence. A striking example is Argentina, where the legal term of office of the central bank governor is four years, but where there it is also an informal tradition that the governor will resign whenever there it is change of government, or even a new finance minister.” (de Haan e Kooi, 2000)

Sob essa visão, uma elevada rotatividade significaria menor nível de independência, pois implicaria um mandato do dirigente do banco central inferior ao do chefe do executivo. Logo, haveria a possibilidade do surgimento de uma dificuldade para a implementação de políticas de longo prazo, tal como a estabilidade de preços. Todavia, conforme reconhecido por Cukierman (1996, p. 396) "A baixa rotatividade não implica necessariamente níveis altos de independência — um presidente relativamente subserviente pode durar no cargo exatamente porque não oferece resistência ao Executivo".

Embora a maior parte da literatura econômica seja favorável à adoção de um banco central independente para a economia, um dos principais problemas concernente a essa proposição refere-se à dificuldade de avaliar os possíveis efeitos sobre a economia devido ao aumento da independência. Outro agravante

\footnotetext{
${ }^{5}$ Ver, por exemplo, Bade e Parkin (1985), Alesina (1989), Grilli, Masciandaro e Tabellini (1991) e Eijffinger e Schaling (1993).
} 
identificado por Hutchison e Walsh (1998) é que grande parte da literatura empírica é resultado de análises de corte transversal sendo considerados na mesma amostra variáveis de diversos países. Portanto, há uma dificuldade em avaliar os efeitos de mudanças institucionais dentro de um determinado país.

Dando continuidade aos estudos que procuram verificar se o aumento da independência do banco central é capaz de reduzir o nível de preços em uma determinada economia, a análise para o caso brasileiro é realizada. O estudo mostrase relevante devido ao fato de que desde o final dos anos 1980 tem sido observado um aumento gradual na independência do Banco Central do Brasil. Ademais, o Brasil obteve sucesso no controle da inflação desde meado dos anos 1990. Portanto, a análise para o caso brasileiro permite avaliar se o aumento observado na independência do banco central ao longo do tempo contribuiu para a obtenção da estabilidade de preços.

\section{O AUMENTO DO GRAU DE INDEPENDÊNCIA}

A economia brasileira ao longo da segunda metade dos anos 1980 e início dos anos 1990 foi marcada pelo descontrole da inflação. Entre 1986 e 1994 foram adotados diversos planos de estabilização (Cruzado, Bresser, Verão, Collor I e Collor II), que se mostraram insuficientes para a tarefa de conter a inflação. Somente depois da introdução do Plano Real (1994) a inflação arrefeceu e atingiu níveis aceitáveis. ${ }^{6}$ Com o objetivo de verificar se o aumento da independência do banco central contribuiu para o controle da inflação foi estimado o grau de independência no período 1980-2002 com base na metodologia desenvolvida por $\mathrm{Cu}$ kierman (1992) e Cukierman, Webb e Neyapti (1992).

Ao contrário dos índices de independência legal — que procuram evidenciar a relação entre a política monetária e as leis que estabelecem e delimitam o poder dos bancos centrais; a metodologia empregada tem como objetivo obter o índice de independência real - que avalia o comportamento da autoridade monetária por meio de um questionário. Deve-se ressaltar que em relação ao comportamento do banco central, a rotatividade dos presidentes é vista como um importante indicador para aferir a independência real dos bancos centrais. A idéia é que no caso de elevada rotatividade o grau de independência seja menor, pois poderia haver uma maior pressão política devido ao fato do mandato do presidente do banco central ser menor do que o do Presidente da República. Ademais, haveria uma maior dificuldade para implementar uma política voltada para a estabilidade de preços devido aos hiatos longos e variáveis entre moeda e preços.

A justificativa para a escolha da metodologia com base em Cukierman (1992)

\footnotetext{
${ }^{6}$ O Plano Real foi um programa de estabilização dividido em três etapas: (i) equilíbrio orçamentário; (ii) introdução de uma nova unidade de conta estável para alinhar os preços relativos mais importantes na economia; e (iii) a conversão desta unidade (URV — unidade real de valor) em nova moeda do país (o Real) com paridade semifixa com o dólar.
} 
e Cukierman, Webb e Neyapti (1992) é devido ao fato de que a literatura sobre o tema ressalta que a utilização de índices de independência legal não se mostra adequada para a análise de países em desenvolvimento. Portanto, ainda que a metodologia apresentada pelos autores sobreditos esteja sujeita a algumas imperfeições, como a possibilidade de haver um viés para uma indicação de independência acima da real, isso não invalida a análise pretendida. A vantagem da metodologia adotada é que ela permite capturar as variações no comportamento da autoridade monetária. Ademais, o efeito da independência do banco central sobre a inflação pode ser observado levando em conta tanto um indicador com base em julgamentos sobre o comportamento (obtido a partir de questionário) quanto do comportamento de fato do banco central (representado pela rotatividade). No caso brasileiro esse tipo de avaliação é importante devido à alteração na postura do Banco Central do Brasil no período posterior à introdução do Plano Real.

A tabela 1 apresenta a rotatividade dos presidentes do Banco Central do Brasil para o período março de 1979 - dezembro de 2002. Desde 1985 observa-se que a rotatividade vem decrescendo. A rotatividade apresentou sua menor magnitude durante o segundo mandato do governo Fernando Henrique apresentando uma redução de $56 \%$ comparado ao período imediatamente anterior.

Seguindo-se a metodologia dos autores supracitados, as perguntas utilizadas para a elaboração do índice de independência do Banco Central do Brasil foram:

(i) Qual a rotatividade do presidente do banco central?

(ii) Qual o nível de restrições ao financiamento do setor público via banco central?

(iii) Na prática, em favor de quem se resolvem os conflitos entre o governo e o banco central?

(iv) Quem determina o orçamento do banco central?

(v) Quem determina os salários dos altos funcionários do banco central e a distribuição de seus lucros?

(vi) Existem metas quantitativas de estoque monetário?

(vii) Existem metas de taxas de juro formais ou informais?

(viii) Qual é a prioridade dada para a estabilidade de preços?

(ix) O banco central funciona como banco de desenvolvimento?

Tabela 1: Rotatividade dos presidentes do Banco Central do Brasil

\begin{tabular}{lcc}
\hline Presidentes da República & Período & Rotatividade \\
\hline J oão Batista Figueiredo & $15 / 03 / 1979-15 / 03 / 1985$ & 0,67 \\
J osé Sarney & $15 / 03 / 1985-15 / 03 / 1990$ & 1,20 \\
Fernando Collor & $15 / 03 / 1990-29 / 12 / 1992$ & 1,07 \\
Itamar Franco & $29 / 12 / 1992-01 / 01 / 1995$ & 1,00 \\
Fernando Henrique Cardoso & $01 / 01 / 1995-01 / 01 / 1999$ & 0,75 \\
Fernando Henrique Cardoso & $01 / 01 / 1999-01 / 12 / 2002$ & 0,33 \\
\hline
\end{tabular}

Nota: A taxa de rotatividade corresponde à freqüência de mudança dos presidentes do banco central durante o mandato do Presidente da República. 
O resultado encontrado pode ser sumariado por meio da tabela 2. Observase que o ano de 1994 representou o maior incremento no grau de independência do Banco Central do Brasil (cerca de 48\%) ao longo do período 1980-2002. Portanto, pode-se dizer que o aumento da independência do banco central brasileiro acentuou-se depois da introdução do Plano Real.

Tabela 2: Estimativas para o grau de independência do BCB

\begin{tabular}{lcccccc}
\hline Período & $1980 / 85$ & $1986 / 89$ & $1990 / 93$ & $1994 / 96$ & $1997 / 99$ & $2000 / 02$ \\
\hline G.l. & 0,25 & 0,35 & 0,46 & 0,68 & 0,71 & 0,76 \\
\hline
\end{tabular}

Nota: G.I. = grau de independência; 0 £ G.I. $£ 1.7$

As principais justificativas para o incremento no grau de independência no período pós-Real podem ser divididas em cinco pontos básicos:

(i) uso de uma variação do regime de câmbio fixo ${ }^{8}$ - esta estratégia para a condução da política econômica foi considerada o principal instrumento para assegurar a estabilidade de preços depois da introdução do Plano Real. O argumento central é que por meio da estrutura implementada foi possível impedir a retomada da inflação devido à restrição imposta sobre os preços dos bens domésticos comercializáveis, ao enfraquecimento da inflação inercial que alimentava salários e preços de bens não-comercializáveis, e à ajuda para a convergência da expectativa inflacionária tendo como referência a inflação do país âncora. Além disso, o arranjo cambial adotado criou um mecanismo de ajuste automático para a oferta de moeda, tendo como conseqüência uma redução no problema de inconsistência temporal para a condução da política monetária. A nova unidade monetária (o Real), além de ter sua emissão limitada por lei, teve como garantia o nível de reservas estrangeiras no mesmo volume em circulação, o que sinalizava aos agentes econômicos a idéia do governo de impedir emissões que viessem dar novo fôlego à inflação.

(ii) redução do uso da receita de senhoriagem — dado que uma receita de senhoriagem elevada denota o financiamento do déficit público via expansão monetária, este seria o caso de um baixo grau de independência do banco central, pois indicaria a subserviência da autoridade monetária ao governo. Por outro lado, uma menor receita de senhoriagem representaria restrições ao financiamento

\footnotetext{
${ }^{7}$ A justificativa do procedimento adotado para a obtenção dos graus de independência ao longo do tempo (submissão do questionário a diversos ex-presidentes do Banco Central do Brasil) encontra-se em de Mendonça (2001).

${ }^{8}$ Com a introdução do Plano Real foram observadas três fases da política cambial: a primeira perdurou até o início de março de 1995, sendo caracterizada pela não-intervenção do banco central no mercado; a segunda fase, que corresponde ao período de março a junho de 1995, foi caracterizada pela implementação do regime de bandas cambiais; por último, a terceira fase é marcada pela maior monitoração da taxa de câmbio, sendo adotado leilões de spread e menores faixas de variação cambial intrabanda.
} 
do governo implicando um maior grau de independência. No ano de 1990 a receita de senhoriagem atingiu seu mais alto nível ( $5 \%$ do PIB) ao passo que no período posterior a 1995 manteve-se abaixo de $0,01 \%$ do PIB, o que por conseguinte, contribuiu para aumentar o grau de independência.

(iii) uso de metas intermediárias - com o Plano Real foram estabelecidas metas quantitativas trimestrais para a base monetária, como forma de minimizar a incerteza dos agentes sobre a possibilidade do governo fazer uso do financiamento inflacionário. De acordo com a Lei n ${ }^{\circ}$ 9.069, de 29 de junho de 1995, que tornou válido os atos praticados com base nas medidas provisórias que estabeleceram as regras do Plano Real, as metas de expansão monetária deixaram, a partir de 1995, de ser fixadas em lei, passando a ser estimadas em programação monetária trimestral elaborada pelo banco central. Esta lei concedeu ao Banco Central do Brasil uma maior liberdade para estabelecer as metas a serem seguidas contribuindo para elevar o grau de independência no período.

(iv) uso de metas para a inflação - essa estratégia para a condução da política monetária possui um nível de transparência mais elevado do que no caso do uso de metas intermediárias. Dado que a maioria dos países que adotou esse mecanismo faz uso de uma meta central com uma banda que permite uma oscilação da inflação para cima e para baixo, isto significa que há limites para a variabilidade da inflação. A conseqüência é a redução do viés inflacionário na condução da política monetária, e por conseguinte, um aumento da independência operacional do banco central. Com a introdução do regime de câmbio flexível em janeiro de 1999, a adoção do regime de metas para inflação em junho do mesmo ano representou uma estrutura que assegurou a preocupação do banco central no controle da inflação.

v) redução na rotatividade dos presidentes do banco central - esta constatação denota um menor risco de inconsistência temporal para a condução da política monetária em seu objetivo prioritário para a estabilidade de preços.

\section{INDEPENDÊNCIA, ROTATIVIDADE E INFLAÇÃO}

Depois de identificar os principais motivos que explicam o aumento da independência no período recente é importante analisar se o maior grau de independência e a queda na rotatividade dos presidentes do Banco Central do Brasil contribuíram de alguma forma para o controle da inflação. A figura 1 mostra a evolução do grau de independência, da rotatividade, e da inflação durante o período 1980-2002. Observa-se que há uma significativa variação no grau de independência, na taxa de rotatividade, e na inflação ao longo do tempo. Destarte, torna-se relevante analisar a relação entre os indicadores de independência e a inflação.

Para analisar a relação entre a independência do banco central e a inflação, foi feita uma análise de regressão (método dos mínimos quadrados) do grau de independência e da rotatividade sobre a inflação (medida pelo IPCA), com base 
em dados anuais para o período 1980-2002. Os dados para o grau de independência (CBBI) e a rotatividade dos presidentes (TOR) do Banco Central do Brasil encontram-se disponíveis nas tabelas 1 e 2 . Dado que vários anos durante a década de 1980 e início dos anos 1990 apresentaram uma inflação anual com quatro ou três dígitos utilizou-se uma série transformada para a inflação. Seguindo a estratégia adotada por Cukierman, Webb e Neyapti (1992) para reduzir a heterocedasticidade do erro e melhorar a eficiência do estimador, a inflação transformada corresponde à divisão entre a taxa anual de inflação e a soma da taxa de inflação mais um. Logo, a taxa de inflação transformada (INFD) possui valores entre 0 e 1. Além disso, devido à mudança estrutural ocorrida com a introdução do Plano Real em 1994 foi introduzida uma variável dummy na forma aditiva ( $\mathrm{D}=0$ antes de 1994 e $\mathrm{D}=1$ a partir de 1994) nas estimações.

Figura 1: Inflação, grau de independência, e rotatividade - 1980/2002)

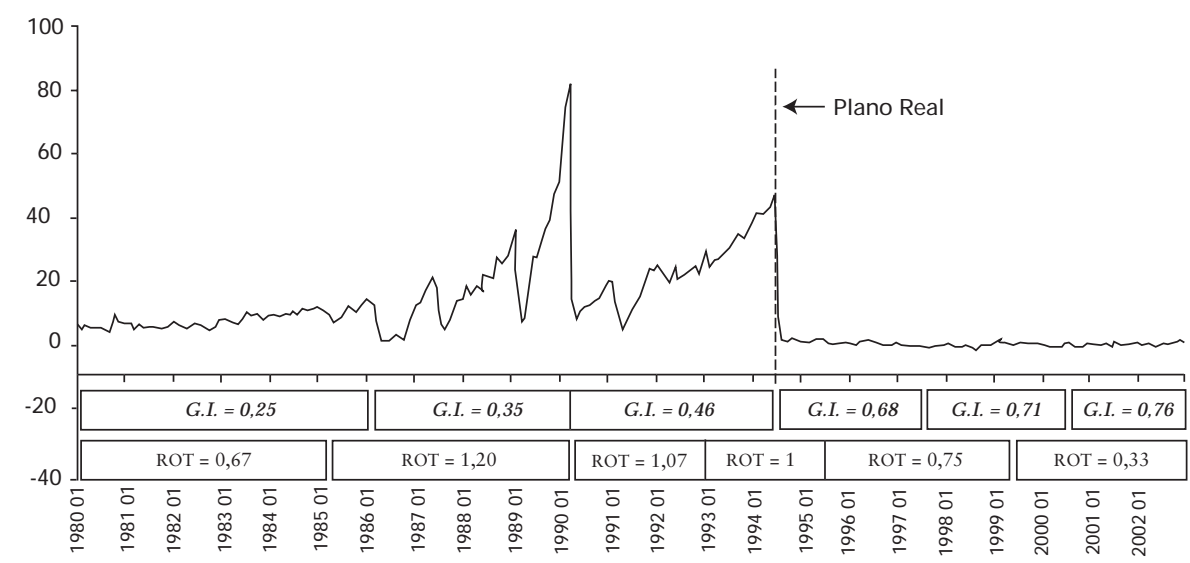

Como forma de verificar se não há espuriedade nos resultados a serem obtidos na regressão por meio do método dos mínimos quadrados é feito o teste de presença de raiz unitária nas séries em consideração. Conforme pode ser observado por meio da tabela 3, tanto o teste ampliado de Dickey-Fuller (ADF) quanto o teste Phillip-Perron (PP) indicam que há aceitação da hipótese nula (séries não estacionárias) para os valores originais das séries CBBI, INFD e TOR. Em relação às primeiras diferenças a hipótese nula é rejeitada para todas as séries ao nível de significância de $1 \%$. Portanto, as três séries são integradas de primeira ordem, isto é, I (1).

Os resultados dos testes de raiz unitária indicam que não é adequado fazer a regressão com as séries em nível. Entretanto, dado que todas as séries são integradas da mesma ordem é possível que haja uma sincronia na tendência das séries. Assim, conforme sugerido por Granger (1986), se as séries forem co-integradas a regressão sobre os níveis das variáveis não é espúria. Com o objetivo de avaliar se as séries são co-integradas foi feito o teste proposto por Johansen (1991). 
Tabela 3: Testes de raiz unitária (ADF e PP)

\begin{tabular}{lcccc}
\hline Séries & Defasagem & Teste ADF & Defasagem & teste PP \\
\hline CBBI & 0 & -2.9531 & 8 & -2.8512 \\
$\Delta$ CBBI & 0 & $-5.3460 *$ & 9 & $-6.0578^{*}$ \\
INFD & 0 & -2.9753 & 1 & -2.9422 \\
$\Delta$ INFD & 0 & $-6.6553^{*}$ & 1 & $-6.6935^{*}$ \\
TOR & 0 & -0.6510 & 1 & -0.6571 \\
$\Delta$ TOR & 0 & $-3.9390^{*}$ & 0 & $-3.9390^{*}$ \\
\hline
\end{tabular}

Nota: Teste ADF — o número de defasagens escolhido leva em conta o critério de Schwarz. Teste PP — número de defasagens (Newey-West utilizando Bartlett kernel). Para as séries CBBI e INFD foram aplicados constante e tendência linear. Para a série $\triangle \mathrm{CBBI}$ foi aplicado constante. Para as demais séries não foi utilizado constante ou tendência.

(*) denota rejeição da hipótese nula de presença de raiz unitária ao nível de significância de 1\%.

Os resultados apresentados para o teste de razão de verossimilhança (teste LR — tabela 4) com base na significância dos autovalores estimados indicam que a estatística do traço ${ }^{9}$ rejeita a hipótese de não co-integração (ao nível de significância de 5\%), mas não a hipótese de que há mais de uma relação de co-integração. Como as séries são co-integradas e, portanto, há uma relação de equilíbrio de longo prazo, isto significa que os resíduos da regressão são estacionários. Logo, os testes t e $\mathrm{R}^{2}$ são válidos e é adequado fazer a regressão pretendida sobre os níveis das variáveis.

Tabela 4: Teste de co-integração de J ohansen

\begin{tabular}{lcccc}
\hline Hipótese nula & Autovalor & Traço & Valor crítico 5\% & Valor crítico 1\% \\
\hline $\mathrm{R}=$ 0* $^{*}$ & 0,684082 & 43,57416 & 42,44 & 48,45 \\
$\mathrm{R} \leq 1$ & 0,427598 & 18,22415 & 25,32 & 30,45 \\
$\mathrm{R} \leq 2$ & 0,236969 & 5,950044 & 12,25 & 16,26 \\
\hline
\end{tabular}

OBS: * denota rejeição de H0 ao nível de significância de 5\%. A especificação selecionada considera tendência e constante na equação co-integrante. Não é aplicada defasagem sobre as séries diferençadas. Os valores críticos foram tabulados por Osterwald-Lenum (1992).

A tabela 5 apresenta os resultados encontrados da regressão do CBBI e TOR sobre a INFD. O coeficiente de determinação indica que as variáveis utilizadas na regressão foram responsáveis por $72 \%$ da variação da inflação transformada. Em relação ao grau de independência observa-se que ao contrário do que é preconizado por grande parte da literatura o sinal mostrou-se positivamente relacionado com a inflação, todavia não houve significância do parâmetro estimado.

\footnotetext{
${ }^{9} \lambda_{\text {traço }}$ - a hipótese nula corresponde ao número de vetores co-integrantes ser igual a r, contra a hipótese alternativa de que o número de vetores co-integrantes é maior que $\mathrm{r}$.
} 
Logo, não houve influência da variável grau de independência na determinação da inflação para o período analisado. Como esperado a variável dummy teve um coeficiente negativo e significativo devido ao sucesso do Plano Real no combate à inflação. Em consonância com a sugestão feita por Cukierman, Webb e Neyapti (1992), o coeficiente de rotatividade dos presidentes do banco central mostrouse positivo e estatisticamente significativo. Portanto, os resultados obtidos denotam a importância da variável rotatividade na explicação da inflação.

Tabela 5: Inflação transformada, grau de independência, e rotatividade

\begin{tabular}{lc}
\hline Variável independente & Inflação transformada (1980-2002) \\
\hline Constante & 0,026 \\
Grau de independência & $(0,116)$ \\
& 0,733 \\
Rotatividade dos presidentes do BCB & $(1,278)$ \\
& 0,472 \\
Dummy: 1994-2002 & $(2,737)^{*}$ \\
& -0.657 \\
N. de observações & $(-2,574)^{*}$ \\
$\mathrm{R}^{2}$ ajustado & 23 \\
\hline
\end{tabular}

Nota: * indica significância ao nível de 1\%. Estatística t entre parênteses.

Com o objetivo de realizar uma análise adicional sobre a causalidade entre a inflação e a rotatividade dos presidentes do Banco Central do Brasil foi feito um teste de precedência temporal de Granger por meio da estimação de um processo autoregressivo. ${ }^{10} \mathrm{O}$ resultado obtido encontra-se na tabela 6 .

Tabela 6: Inflação transformada e rotatividade

\begin{tabular}{lcc}
\hline Variável independente & Inflação transformada & Rotatividade \\
\hline Constante & $-0,028$ & $-0,107$ \\
& $(-0,205)$ & $(0,982)$ \\
Inflação transformada (-1) & 0,799 & 0,223 \\
& $(4,425)^{*}$ & $(1,540)^{* *}$ \\
Rotatividade dos presidentes do BCB (-1) & 0,139 & 0,715 \\
\hline
\end{tabular}

\footnotetext{
${ }^{10} \mathrm{O}$ teste de Granger avalia se uma série de tempo $X_{t}$ "causa” outra série $Y_{t}$, se esta for prevista de forma mais adequada pelos valores passados de $X_{t}$, e por outras variáveis relevantes, inclusive valores passados do próprio $Y_{t}$.
} 
Nota: * e** indicam significância ao nível de 1\% e 10\% respectivamente. Estatística t entre parênteses.

O coeficiente estimado da rotatividade defasada na equação para a inflação transformada não é estatisticamente diferente de zero. Por outro lado, o coeficiente estimado da inflação na equação para a rotatividade é estatisticamente significativo. Em outras palavras, há causalidade unidirecional da inflação para a rotatividade. Este resultado é antagônico ao encontrado na regressão anterior. Logo, não é verdadeira a afirmação de que a queda na rotatividade dos presidentes do Banco Central do Brasil contribuiu para diminuir a inflação no período analisado.

\section{CONCLUSÃO}

Deve-se ressaltar que a metodologia empregada indicou um aumento significativo na independência e queda na rotatividade dos presidentes do banco central depois da introdução do Plano Real. Conforme ressaltado por Cukierman (1996), as informações extraídas dos indicadores de independência devem ser suplementados pela crítica no contexto do problema em consideração. Assim, é importante observar que os elementos-chave para a melhora no perfil dos indicadores de independência são fruto da própria decisão do governo, devido, por exemplo, à preocupação com a estabilidade de preços e o equilíbrio fiscal. Portanto, o próprio governo foi responsável pela criação de um ambiente no qual o Banco Central do Brasil coopera para a obtenção do equilíbrio macroeconômico. Em suma, como os indicadores utilizados baseiam-se no comportamento da autoridade monetária, a introdução do Plano Real foi a responsável por uma mudança qualitativa nos indicadores de independência.

A falta de significância estatística do grau de independência do banco central e da rotatividade sobre a inflação brasileira referente ao período 1980-2002 reflete a observação acima. O aumento da independência e a queda na rotatividade dos presidentes do banco central, obtidos a partir da metodologia utilizada, não representam uma condição básica para a manutenção da estabilidade de preços. De outra forma, pelo menos durante o processo de construção de credibilidade na condução da política econômica, o cenário macroeconômico resultante da implementação do Plano Real é o responsável pela relativa estabilidade de preços.

O resultado empírico obtido é importante porque países que, como o Brasil, buscam credibilidade, devem procurar outros caminhos ao invés de partir do pressuposto de que há um círculo vicioso entre a inflação e a falta de independência do banco central. A conclusão apresentada por Cukierman (1992) de que um banco central independente não representa condição nem necessária nem su- 
ficiente para garantir a estabilidade de preços, e repetida diversas vezes pelos teóricos que discutem o assunto, é, mais uma vez, merecedora de destaque.

\section{REFERÊNCIAS BIBLIOGRÁFICAS}

ALESINA, A. (1989) "Politics and Business Cycles in Industrial Democracies". Economic Policy, 57-98.

ALESINA, A. e SUMMERS, L. (1993) "Central Bank Independence and Macroeconomic Performance: Some Comparative Evidence.” Journal of M oney, Credit and Banking, May, 151-62.

BADE, R. e PARKIN, M. (1985) “Central Bank Lows and Monetary Policy.” Department of Economics, University of Western Ontario.

BERGER, H., de HAAN, J. e EIJFFINGER, S.C.W. (2001) “Central bank independence: an update of theory and evidence." Journal of Economic Surveys, Vol. 15, N. 1, 3-39.

BLINDER, A.S. (2000) "Central-bank credibility: why do we care?" The A merican Economic Review, December, Vol. 90, N. 5, 1421-31.

CUKIERMAN, A. (1996) “A economia do Banco Central.” Revista Brasileira de Economia, Rio de Janeiro, 50(4), out./dez, 389-426.

CUKIERMAN, A. (1992) “Central Bank Strategy, Credibility, and Independence: Theory and Evidence." Cambridge, Mass.: The MIT Press.

CUKIERMAN, A., WEBB, S., e NEYAPTI, B. (1992) “Measuring the Independence of Central Banks and Its Effects on Policy Outcomes." World Bank Economic Review, 6(3), 353-98.

de HAAN, J., e KOOI, W. (2000) “Does Central Bank Independence Really Matter? New Evidence for Developing Countries Using a New Indicator.” Journal of Banking and Finance, 24, 643-64.

de MENDONÇA, H.F. (2002) “A teoria da credibilidade da política monetária: desdobramentos do debate regras versus discrição.” Revista de Economia Política, V. 22, N. 3 (87), julho-setembro, 46-64.

de MENDONÇA, H.F. (2001) “A mensuração do grau de independência do Banco Central: uma análise de suas fragilidades.” A nálise Econômica. Faculdade de Ciências Econômicas, UFRGS, Ano 19, N. 36, setembro, p. 47-67.

de MENDONÇA, H.F. (2000) “A teoria da independência do Banco Central: uma interpretação crítica.” Estudos Econômicos, Instituto de Pesquisas Econômicas - USP, São Paulo, V. 30, N. 1, 101-27.

EIJFFINGER, S., e SCHALLING, E. (1993) “Central Bank Independence in Twelve Industrial Countries.” Banca N azionale del L avoro Q uarterly Review, N. 184, March, 49-89.

FISCHER, S. (1995) “Central Bank Independence Revisited.” A merican Economic R eview, V. 85, N. 2, May, 201-06.

GOODHART, C.A.E. (1994) “Game Theory for Central Bankers: a Report to the Governor of the Bank of England.” Journal of E conomic Literature, March, 32, 101-14.

GRANGER, C.W.J. (1986) "Developments in the Study of Co-integrated Economic Variables." Oxford Bulletin of E conomics and Statistics, V. 48.

GRILLI, V.; MASCIANDARO, D.; e TABELLINI, G. (1991) “Political and Monetary Institutions and Public Financial Policies in the Industrial Countries.” E conomic Policy (13), 341-92.

HUTCHISON, M.M. e WALSH, C.E. (1998) “The output-inflation tradeoff and central bank reform: evidence from New Zealand.” The Economic Journal, Vol. 108, May, 703-25.

JOHANSEN, S. (1991) "Estimation and Hypothesis Testing of Cointegration Vectors in Gaussian Vector Autoregressive Models,” Econometrica, 59, 1551-80.

OSTERWALD-LENUM, M. (1992) “A Note with Quantiles of the Asymptotic Distribution of the Maximum Likelihood Cointegration Rank Test Statistics," O xford Bulletin of Economics and Statistics, 54, 461-72.

POSEN, A. (1998) “Central Bank Independence and Disinflationary Credibility: a Missing Link.” O xford Economic Papers, V. 50, N. 3, July, 335-59. 